\title{
Passing the "Acid Test": Do Proton Pump Inhibitors Affect the Composition of the Microbiome?
}

\author{
Tien Dong ${ }^{1} \cdot$ Joseph Pisegna ${ }^{2,3}$
}

Published online: 14 September 2018

(c) This is a U.S. Government work and not under copyright protection in the US; foreign copyright protection may apply 2018

With the rising prevalence of gastroesophageal reflux disease (GERD) and other acid-peptic diseases, proton pump inhibitors (PPIs) are among the top 10 most commonly used medication worldwide [1]. While PPI use is of considerable value in the treatment of Helicobacter pylori infection and peptic ulcer disease, in recent years, chronic PPI use has also been linked to $C$. difficile infection, small intestinal bacterial overgrowth, and alterations of the intestinal microbiome [2]. The gut microbiome has been considered to be important for the maintenance of gut homeostasis and may even protect against infection with enteric organisms [3]. Though antibiotics are the most common drug class associated with gut microbial community shifts, there has been an increasing level of evidence that links nonantibiotic drugs to microbiome changes [4]. For this reason, there have been many studies of late examining the effect of PPI use on the composition of the gut microbiome.

There are at least two known mechanisms by which PPI use can affect the composition of microbial communities: by directly targeting bacterial proton pumps and by altering the gut microenvironment as a consequence of inhibiting gastric acid secretion $[1,5]$. In a cohort of 211 patients, PPI use was associated with a significant decrease in microbial diversity and an increase in the abundance of several genera such as Enterococcus, Streptococcus, Staphylococcus and even potentially pathogenic species of $E$. Coli [2]. In recent metanalyses, PPI use was associated with a significant increase

Joseph Pisegna

Joseph.Pisegna@va.gov

1 The Vatche and Tamar Manoukian Division of Digestive Diseases, Department of Medicine, David Geffen School of Medicine at UCLA, Los Angeles, USA

2 Division of Gastroenterology, Hepatology and Parenteral Nutrition, Department of Medicine, VA Greater Los Angeles Healthcare System, Los Angeles, USA

3 Department of Human Genetics, David Geffen School of Medicine at UCLA, 200 UCLA Medical Plaza, Suite 365-A, Los Angeles, CA 90095, USA in Streptococcus and Enterococcus [6]. These changes were associated with an increased risk for the development of spontaneous bacterial peritonitis, community-acquired pneumonia, and enteric infections. Nevertheless, the exact mechanism by which these microbial community alterations affect disease susceptibility is still an active area of investigation.

In the current issue of Digestive Diseases and Sciences, Hojo et al. [7] performed a novel clinical study examining the changes in fecal microbiota composition, fecal shortchain fatty acids (SCFA) content, and changes in bloodborne gut microbes before and after PPI use. Twenty patients with endoscopic evidence of GERD were given 8 weeks of PPI therapy. Patients were excluded if they had previously undergone gastrointestinal resection or if they were already receiving PPI or antibiotic therapy, or were taking probiotics 1 month prior to enrollment. By using targeted real-time quantitative polymerase chain reaction (RT-qPCR) and metabolomic analysis, Hojo et al. found that PPI use increased the abundance of Lactobacillus species as compared to baseline. Specifically, they found an increase in $L$. gasseri, L. fermentum, L. reuteri, L. ruminis, and L. brevis. Similar to previous larger cohorts that used 16S rRNA next-generation sequencing, they also saw an increase in Streptococcus, Staphylococcus, and Enterococcus. This shift was associated with a concomitant increase in formic acid and butyric acid without any significant differences in blood bacterial count. This study corroborates prior studies that reported similar increases in Streptococcus and Enterococcus species. [1, 6] Since the species that were differentially increased are those often associated with the oral microbiome, the authors suggested that the increase in gastric $\mathrm{pH}$ as a consequence of PPI therapy may have increased bacterial migration from the oral cavity to the intestinal lumen through decreased gastric acid-related bacterial killing.

The main strengths of the study include their novel use of targeted RT-qPCR, analysis of fecal SCFA, and quantification of bacteria in the blood. The use of the proprietary Yakult Intestinal Flora-SCAN (YIF-SCAN ${ }^{\circledR}$ ) RT-qPCR 
platform enables high sensitivity and high resolution of bacterial species and subdominant populations. Even though it gives a limited coverage of bacterial genera, it is a more sensitive approach for specific species than is 16S metagenomics. Furthermore, the analysis of SCFA may inform a mechanistic explanation regarding how microbial changes can affect the host. Luminal SCFA, the products of bacterial metabolism of undigested carbohydrates, have been linked to differential gene regulation, decreased intestinal paracellular permeability, the release of gut hormones such as glucagon-like peptides 1 and 2, and altered host metabolism $[8,9]$. Nevertheless, alteration of fecal SCFA amounts only occurred after 4 but not after 8 weeks of PPI therapy. The authors also innovatively measured bacterial counts in the blood as a surrogate for intestinal permeability and bacterial translocation. Even though they did not reach significance, there was a trend toward higher bacterial counts and higher detection of Streptococcus in the blood 8 weeks post-PPI therapy.

Even though there were several interesting findings, there are several weaknesses in the paper. The first weakness is the study's small sample size. Although there were interesting trends such as increased total bacterial blood count and median Streptococcus blood count 8 weeks post-PPI therapy, the study's small sample size limited their ability to reach significance. Furthermore, the concept of bacterial translocation from the gut to the blood in normal healthy humans is still controversial and the use of PCR to detect blood-borne commensal species has not yet been validated. The study, while informative, was still based mostly on fecal microbial changes associated with short-term PPI use. There are very few studies that exist that are able to correlate the composition of the fecal microbiome to luminal or mucosa-associated microbial communities, and few would suggest that their compositions can be quite different $[10,11]$. Due to this, the author's main conclusions of fecal microbial changes are merely speculative to the changes possibly seen within the gastric lumen or mucosa. Without directly measuring gastric $\mathrm{pH}$ or gastric luminal contents, the authors are also unable to support if the changes noted were due to a significant decrease in gastric $\mathrm{pH}$ due to PPI therapy. Lastly, the pathophysiological significance of these microbial changes was not explored and the study's short duration limited the author's ability to correlate these findings with more clinically relevant endpoints such as the incidence of enteric infections. But in a recent age-sex-matched case-control study of 36 chronic PPI users compared to 36 controls, similar increases in Streptococcus were observed in chronic PPI users, suggesting that the observed short-term changes persist with chronic users [12].

Future studies should focus on developing plausible hypotheses regarding how PPI therapy affects the host. Measurement of fecal microbial composition and metabolite concentrations in and of itself provides little information regarding why alterations of the gut microbiome are associated with clinical outcomes such as increased infection susceptibility. Advancing beyond metagenomic analysis to how bacterial metabolites such as SCFAs and bacterial products such as lipopolysaccharide interact with specific host receptors expressed on key cell types such as enteroendocrine, pro-inflammatory, neural, and immune cells will likely provide fresh insight into the pathobiology of the microbiomehost relationship. If an increased gastric $\mathrm{pH}$ was the main mechanism by which the observed changes occurred, then a prospective study in which gastric $\mathrm{pH}$ was noninvasively measured could then be correlated with bacterial dysbiosis and infection susceptibility.

In summary, Hojo et al. provided interesting insights relating PPI use to fecal microbial composition and SCFA concentrations, and possibly to bacterial translocation from lumen to blood. Given the widespread use of PPIs, the morbidity related to enteric infections and the ever-increasing number of studies examining the microbiome, healthcare providers and researchers should be fully cognizant of the potential effects of long-term PPI therapy on human health.

\section{References}

1. Minalyan A, Gabrielyan L, Scott D, Jacobs J, Pisegna JR. The gastric and intestinal microbiome: role of proton pump inhibitors. Curr Gastroenterol Rep. 2017;19:42. https://doi.org/10.1007/ s11894-017-0577-6.

2. Imhann F, Bonder MJ, Vich Vila A, et al. Proton pump inhibitors affect the gut microbiome. Gut. 2016;65:740-748. https:// doi.org/10.1136/gutjnl-2015-310376.

3. Buffie CG, Pamer EG. Microbiota-mediated colonization resistance against intestinal pathogens. Nat Rev Immunol. 2013;13:790801. https://doi.org/10.1038/nri3535.

4. Maier L, Pruteanu M, Kuhn M, et al. Extensive impact of nonantibiotic drugs on human gut bacteria. Nature. 2018;555:623628. https://doi.org/10.1038/nature25979.

5. Vesper BJ, Jawdi A, Altman KW, Haines GK, Tao L, Radosevich JA. The effect of proton pump inhibitors on the human microbiota. Curr Drug Metab. 2009;10:84-89.

6. Naito Y, Kashiwagi K, Takagi T, Andoh A, Inoue R. Intestinal dysbiosis secondary to proton-pump inhibitor use. Digestion. 2018;97:195-204. https://doi.org/10.1159/000481813.

7. Hojo M, Asahara T, Nagahara A, et al. Gut microbiota composition before and after use of proton pump inhibitors. Dig Dis Sci. (Epub ahead of print). https://doi.org/10.1007/s1062 0-018-5122-4.

8. Morrison DJ, Preston T. Formation of short chain fatty acids by the gut microbiota and their impact on human metabolism. Gut Microbes. 2016;7:189-200. https://doi.org/10.1080/19490 976.2015.1134082.

9. Tan J, McKenzie C, Potamitis M, Thorburn AN, Mackay CR, Macia L. The role of short-chain fatty acids in health and disease. Adv Immunol. 2014;121:91-119. https://doi.org/10.1016/B9780-12-800100-4.00003-9. 
10. Bajaj JS, Hylemon PB, Ridlon JM, et al. Colonic mucosal microbiome differs from stool microbiome in cirrhosis and hepatic encephalopathy and is linked to cognition and inflammation. Am J Physiol Gastrointest Liver Physiol. 2012;303:G675-G685. https ://doi.org/10.1152/ajpgi.00152.2012.

11. Zoetendal EG, von Wright A, Vilpponen-Salmela T, Ben-Amor $\mathrm{K}$, Akkermans ADL, de Vos WM. Mucosa-associated bacteria in the human gastrointestinal tract are uniformly distributed along the colon and differ from the community recovered from feces. Appl Environ Microbiol. 2002;68:3401-3407.

12. Takagi $T$, Naito $Y$, Inoue $R$, et al. The influence of long-term use of proton pump inhibitors on the gut microbiota: an age-sexmatched case-control study. J Clin Biochem. 2018;62:100-105. https://doi.org/10.3164/jcbn.17-78. 